\title{
New Greener Alternative for Biocondensation of Aldehydes and Indoles Using Lemon Juice: Formation of Bis-, Tris-, and Tetraindoles
}

\author{
Rammohan Pal \\ Department of Chemistry, Acharya Jagadish Chandra Bose College, Kolkata, India \\ Email:pal_rammohan@yahoo.com
}

Received March 30, 2013; revised May 6, 2013; accepted May 20, 2013

Copyright (C) 2013 Rammohan Pal. This is an open access article distributed under the Creative Commons Attribution License, which permits unrestricted use, distribution, and reproduction in any medium, provided the original work is properly cited.

\begin{abstract}
This work presents a new greener alternative for biocondensation of aldehydes and indoles for the synthesis of bis- and tris(indolyl)methanes catalyzed by lemon juice (Citrus limon) in good yields under ultrasound irradiation in aqueous ethanol. Various substituted aldehydes with indoles under this reaction condition are elucidated. Also, tetraindolyl compounds were prepared using terephthaldialdehyde by following the same protocol. This method is an environmentally benign, efficient reaction, which requires shorter reaction time and simple experimental and work-up procedures.
\end{abstract}

Keywords: Citrus limon Juice; Biocondensation; Aldehydes; Indoles; Aqueous Ethanol; Sonication; Bis-; Tris-; Tetraindoles

\section{Introduction}

Indoles and their derivatives are known as an important class of heterocyclic compounds and bioactive intermediates in $\mathrm{R} \& \mathrm{D}$ and pharmaceutical industry [1]. Bis(indolyl)methanes (BIMs) and tris(indolyl)methanes (TIMs) exhibit a wide range of biological activities [2]. Tetraindolyl compounds such as 1,4-di[bis(3-indolyl)methyl]benzene are used in the treatment of fibromyalgia, chronic fatigue and irritable bowel syndrome [3]. Due to the versatile applications of BIMs and TIMs, there is continuous interest in the synthesis of these compounds [4]. Synthetically, the reaction of indole with aldehyde or ketone produces azafulvenium salts that react further with a second indole molecule to form bis(3-indolyl)methanes [5]. Different protic acids [6,7] including silica sulfuric acid [8], silica supported $\mathrm{NaHSO}_{4}$ and amberlyst-15 [9], silica supported $\mathrm{KaHSO}_{4}$ [10] and Lewis acids [2,11-13] are known to catalyze this reaction. The protic acids (e.g., $\mathrm{HCl}$ and $\mathrm{H}_{2} \mathrm{SO}_{4}$ ) and Lewis acids (e.g., $\mathrm{BBr}_{3}$ and $\mathrm{BF}_{3}$ ) which are generally used are hazardous and difficult to handle and remove from the reaction mixture. Some of the reported catalysts may decompose the indoles or disturb the functionalities. Several catalysts are not also readily available or expensive. Recently, the reactions were carried in acetonitrile in presence of many kind of catalyst, such as $\mathrm{InCl}_{3}, \mathrm{In}(\mathrm{OTf})_{3}[14], \mathrm{I}_{2}$
[15], trichloro-1,3,5-triazine (TCT) [16], $\mathrm{PPh}_{3} \cdot \mathrm{HClO}_{4}$ [17]. However, the acetonitrile is not an environmentally friendly solvent and therefore environmentally friendly and cheaper solvent is desirable.

Organic reactions in aqueous media or aqueous ethanol media have attracted much attention because both water and ethanol are considerably safe, non-toxic, environmentally-friendly, and cheap compared to organic solvents [18]. The synthesis of bis(indolyl)methanes in water was first reported using acetic acid [19] at $\mathrm{pH} 2.5$ at room temperature for 10 days. Subsequent methods for the reaction in water have been reported using alum [20], benzoic acid [21], in aqueous ethanol using lanthanide triflate [22], zirconium oxychloride [23]. The use of surfactant or ionic liquid such as sodium dodecyl sulfate (SDS) [24], oxalic acid/ $N$-acetyl- $N, N, N$-trimethylammonium bromide (CTAB) [25] and ferric dodesyl sulfonate $\left[\mathrm{Fe}(\mathrm{DS})_{3}\right][26]$ has also been reported. Most of these methods suffer from various disadvantages such as long reaction periods, harsh experimental procedure and surfactants or ionic liquid that are expensive.

Ultrasound has increasingly been used in organic synthesis in the last three decades. Compared with traditional methods, the ultrasound procedure is more convenient and can be carried out in higher yield, shorted reaction time or mild conditions [27,28]. Zeng, et al. [29] 
have reported synthesis of BIMS catalyzed by ceric ammonium nitrate (CAN) under ultrasound irradiation. Subsequently, other ultrasound irradiation methods using aminosulfonic acid [30], meldrum's acid [31], silicotungstic acid [32] and acrylonitrile butadiene styrene (ABS) [33] were also reported. However, the catalysts used are hazardous, expensive and not easily available.

Among the challenges for chemists including discovery and development of inexpensive, non-hazardous and simple environmentally safe chemical processes for selective synthesis by identifying alternative reaction conditions and solvents that much improved selectivity, energy conservation and even less hazardous waste generation are not desirable and inherently safer chemical products. Therefore, to address depletion of natural resources and preservation of ecosystem is just urgent to develop so called "greener technologies" to make chemical agents for well being of human heath. Due to acidic nature $(\mathrm{pH}=3.0)$, aqueous Citrus limon juice as a natural catalyst has been found to be a suitable replacement for various homogeneous acid catalysts.

\section{Results and Discussion}

In continuation of our research and interest in the developement of novel synthetic methodologies using fruit juice $[34,35]$, herein we would like to report an one-pot two component synthesis of bis-, tris-, and tetra-(indolyl)methanes using Citrus limon juice as natural catalyst. The biocondensation between indoles and aldehydes takes place smoothly in presence of Citrus limon juice in aqueous ethanol under ultrasound irradiation without the use of any surfactant. The juice of the Citrus limon contains citric acid $(5 \%-8 \%)$ and ascorbic acid are the main components of the juice which acts as the effective acid catalyst by activating the carbonyl group of the aldehydes in this reaction. The juice is highly soluble in water and thus acts as a homogeneous catalysis on biocondensation of indoles and aldehydes in aqueous ethanol system. Ethanol is also a green solvent used along with water because aldehydes and indoles is less soluble in water only.

We have recently observed that Citrus limon juice acts a homogeneous acid catalyst for the electrophilic substitution reaction of indoles with aldehydes (Scheme 1). We have found that indole or 2-methylindole $(2 \mathrm{mmol})$ when reacted with various aldehydes $(1 \mathrm{mmol})$ in presence of Citrus limon juice $(2 \mathrm{ml})$ in ehthanol-water mixture $(8 \mathrm{ml}$, ratio: $3: 5)$ at $60^{\circ} \mathrm{C}$ under ultrasound irradiation produces bis(3-indolyl)methanes (3a-x) in high yields (Scheme 1, Table 1).

The scope of application of the presented method is demonstrated by using the various substituted aromatic and aliphatic aldehydes to react with indoles. The proce- dure was successfully applied for heteroaromatic aldehyde (entry $\mathrm{n}$ ) and the corresponding BIMs was obtained in $85 \%$ yield. The electronic nature of the substituents in the aromatic ring did not show any noticeable effect on this conversion. The long-chain aliphatic aldehydes (entries $\mathrm{o}, \mathrm{p}$ and $\mathrm{x}$ ) also worked well to form the products in high yields. The ether (entries $b, d, e, f, r, t$ and $v$ ) and esters linkages (entries $f, h$ and $v$ ) present in the aldehydes were unaffected. The activity of indole and 2-methylindole was found to be almost similar towards the reaction.

The reaction was further explored for the synthesis of tris(3-indolyl)methanes by the condensation of an heteroaromatic aldehyde i.e. 3-formylindole with two equivalents of indoles under similar condition at $60^{\circ} \mathrm{C}$. The products $5 \mathrm{a}$ and $5 \mathrm{~b}$ were produced in $60 \%$ and $75 \%$ yields respectively (Scheme 2, Table 2).

Interstingly, Citrus limon juice was effectively used for the synthesis of di-bis(indolyl)methanes from indoles and terephthaldialdehyde (6) (Scheme 3, Table 2). The reaction of 2 equivalents of indoles with 1 equivalent of terephthaldialdehyde proceeded successfully to give $p$ -

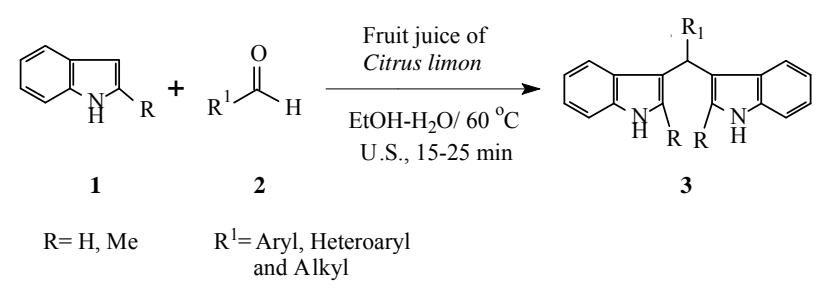

Scheme 1. Synthesis of bis(3-indolyl)methanes.

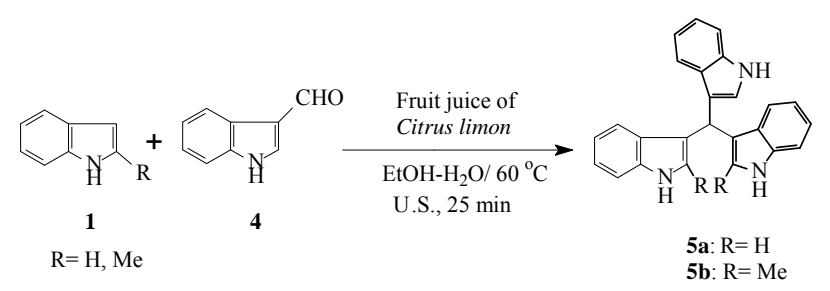

Scheme 2. Synthesis of tris(3-indolyl)methanes.

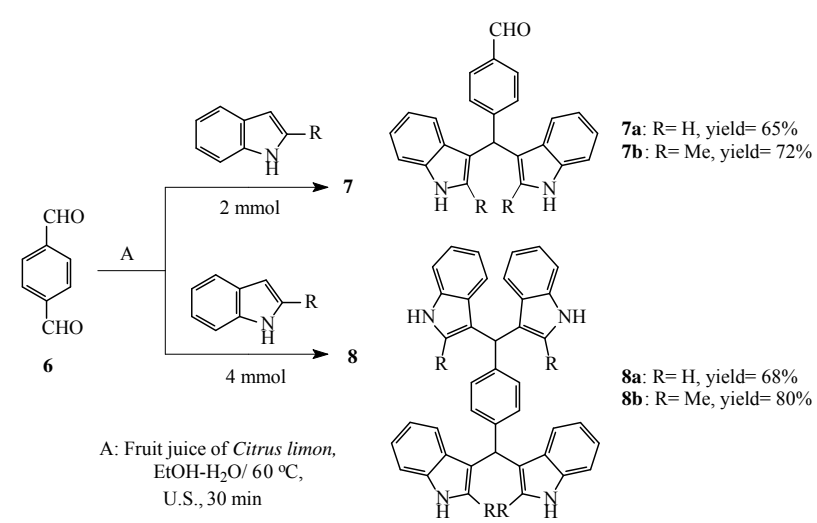

Scheme 3. Synthesis of $p$-bis(indolyl)methyl benzaldehydes and tetraindoles. 
Table 1. Synthesis of bis(3-indolyl)methanes (3) from the biocondensation of indoles (1) with aldehydes (2) using fruit juice of Citrus limon under ultrasound irradiation ${ }^{\mathrm{a}}$.

\begin{tabular}{|c|c|c|c|c|c|c|c|}
\hline Entry & R (1) & $\mathbf{R}^{1}$ (2) & Product (3) & Time (min) & Yield (\%) & M.p. $\left({ }^{\circ} \mathrm{C}\right)$ (Lit. Value) & References \\
\hline a & $\mathrm{H}$ & & $3 \mathbf{a}$ & 15 & 80 & $86-88(88-90)$ & 13 \\
\hline $\mathrm{b}$ & $\mathrm{H}$ & & $3 \mathbf{b}$ & 20 & 75 & $183-184(185-187)$ & 32 \\
\hline $\mathrm{c}$ & $\mathrm{H}$ & & 3c & 18 & 80 & 93 - 95 (94 - 96) & 15 \\
\hline $\mathrm{d}$ & $\mathrm{H}$ & & 3d & 20 & 82 & 95 - 96 (97 - 99) & 13 \\
\hline $\mathrm{e}$ & $\mathrm{H}$ & & $3 \mathbf{e}$ & 25 & 72 & $125(126-127)$ & 24 \\
\hline $\mathrm{f}$ & $\mathrm{H}$ & & $3 f$ & 17 & 85 & $238-240(240-241)$ & 34 \\
\hline g & $\mathrm{H}$ & & $3 g$ & 23 & 80 & $121(120-121)$ & 25 \\
\hline $\mathrm{h}$ & $\mathrm{H}$ & & $3 \mathbf{h}$ & 15 & 88 & $216(218-220)$ & 34 \\
\hline $\mathrm{i}$ & $\mathrm{H}$ & & $3 \mathbf{i}$ & 15 & 78 & $73-74(74-76)$ & 12 \\
\hline $\mathrm{j}$ & $\mathrm{H}$ & & $3 \mathbf{j}$ & 20 & 70 & $108-111(110-112)$ & 25 \\
\hline $\mathrm{k}$ & $\mathrm{H}$ & & $3 \mathbf{k}$ & 15 & 90 & $220(220-222)$ & 32 \\
\hline 1 & $\mathrm{H}$ & & $3 \mathbf{l}$ & 15 & 95 & $217-219(219-222)$ & 12 \\
\hline $\mathrm{m}$ & $\mathrm{H}$ & & $3 m$ & 25 & 70 & $208-210(210-212)$ & 25 \\
\hline $\mathrm{n}$ & $\mathrm{H}$ & & $3 n$ & 25 & 80 & $321-323(322-324)$ & 25 \\
\hline o & $\mathrm{H}$ & & 30 & 20 & 80 & $107(106-108)$ & 11 \\
\hline $\mathrm{p}$ & $\mathrm{H}$ & & $3 p$ & 20 & 81 & $67-69(68-70)$ & 13 \\
\hline q & $\mathrm{Me}$ & & $3 q$ & 15 & 76 & $245-246(247-248)$ & 24 \\
\hline $\mathrm{r}$ & $\mathrm{Me}$ & & $3 r$ & 20 & 70 & $98-99(98-100)$ & 13 \\
\hline $\mathrm{s}$ & $\mathrm{Me}$ & & $3 s$ & 18 & 85 & $173-174(174-175)$ & 24 \\
\hline $\mathrm{t}$ & $\mathrm{Me}$ & & $3 t$ & 20 & 77 & $225-227(226-228)$ & 21 \\
\hline $\mathrm{u}$ & $\mathrm{Me}$ & & $3 \mathbf{u}$ & 15 & 83 & $236-238(238-239)$ & 21 \\
\hline $\mathrm{v}$ & $\mathrm{Me}$ & & $3 \mathbf{v}$ & 15 & 90 & $275-277(274-275)$ & 34 \\
\hline $\mathrm{w}$ & $\mathrm{Me}$ & & $3 w$ & 20 & 70 & $239(241-243)$ & 35 \\
\hline $\mathrm{x}$ & $\mathrm{Me}$ & & $3 x$ & 20 & 73 & $127(129-130)$ & 34 \\
\hline
\end{tabular}

${ }^{a}$ All reactions were carried out with $2 \mathrm{mmol}$ of indole, $1 \mathrm{mmol}$ of aldehyde, $2 \mathrm{ml}$ Citrus limon juice, $5 \mathrm{ml}$ water and $3 \mathrm{ml}$ ethanol at $\mathrm{pH}=3$ at $60^{\circ} \mathrm{C}$; ${ }^{b} \mathrm{Yield}$ of the isolated product after chromatography on silica gel. 
Table 2. Citrus limon juice catalyzed synthesis of tris(indolyl)methanes (5), p-bis(indolyl)methyl benzaldehydes (7) and tetraindoles (8).

\begin{tabular}{|c|c|c|c|c|c|c|}
\hline \multirow{2}{*}{ Entry } & \multirow{2}{*}{ Product } & \multirow{2}{*}{ Time (min) } & \multirow{2}{*}{ Yield (\%) } & \multicolumn{2}{|c|}{ Melting. Point. $\left({ }^{\circ} \mathrm{C}\right)$} & \multirow{2}{*}{ References } \\
\hline & & & & Found & Reported & \\
\hline a & $5 a$ & 25 & 60 & $246-248$ & $249-251$ & 35 \\
\hline $\mathrm{b}$ & $5 \mathbf{b}$ & 25 & 75 & $264-267$ & $268-269$ & 35 \\
\hline $\mathrm{c}$ & $7 a$ & 25 & 65 & $254-256$ & $253-255$ & 10 \\
\hline d & $7 \mathbf{b}$ & 25 & 72 & $230-232$ & $232-234$ & 35 \\
\hline $\mathrm{e}$ & $8 a$ & 30 & 68 & $192-194$ & $194-195$ & 25 \\
\hline $\mathrm{f}$ & $8 b$ & 30 & 80 & $282-285$ & $280-280$ & 35 \\
\hline
\end{tabular}

bis(indolyl)methane benzaldehydes (7a-b) in good yields. On the other hand, using 4 equivalents of indoles, $p$ di(bis-indolylmethane)benzenes (8a-b) were obtained in high yields within 30 minutes under similar reaction conditions.

All the products are known compounds and their structures are settled by comparison of melting points and spectral (IR, ${ }^{1} \mathrm{H}$ NMR and Mass) data with their literature data.

\section{Conclusion}

We have developed an eco-friendly and economic process for the synthesis of bis-, tris-, and tetraindoles by Citrus limon juice as a natural catalyst with good yields in aqueous ethanol at $\mathrm{pH}=3$ at $60^{\circ} \mathrm{C}$ under ultrasound irradiation. This approach is totally nonpolluting; there is no use of toxic materials, quantifying it is a green approach to this biocondensation reaction between indoles and aldehydes.

\section{Experimental Section}

\subsection{Preparation of Lemon Juice from the Fruits of Citrus Limon}

Fresh fruits of Citrus limon was purchased from the local market. The fruit's juice was extracted mechanically and centrifuged using Micro Centrifuge (REMI RM-12C). The clear portion of the juice was used as catalyst for the reactions.

\subsection{General Procedure for Biocondensation of Indoles and Aldehydes by Lemon Juice}

Indoles (2 mmol), aldehydes (1 mmol), fruit juice of Citrus limon $(2 \mathrm{ml})$, ethanol $(3 \mathrm{ml})$, and water $(5 \mathrm{ml})$ were mixed in a $50 \mathrm{ml}$ round bottomed flask. The reaction mixture $(\mathrm{pH}=3)$ was irradiated in water bath of an ultrasonic cleaner (RIVOTEK with a frequency of 30 $\mathrm{kHz}$ and a nominal power $50 \mathrm{~W}$; Riviera Glass Pvt. Ltd.) at $60^{\circ} \mathrm{C}$ for the period indicated in Table 1. After completion of the reaction, the reaction mixture was diluted with water $(5 \mathrm{ml})$ and extracted three times with ethyl acetate $(3 \times 10 \mathrm{ml})$. The combined organic layers were dried over anhydrous sodium sulphate and filtered. And then the solvent from the extract was evaporated under reduced pressure to give the residue, which was purified by column chromatography over silica gel (100 - 200 mesh) using mixtures of petroleum ether and ethyl acetate as eluent to afford the bis(3-indolyl)methanes (3, Scheme 1, Table 1) which was finally crystallized from ethyl acetate-petroleum ether. The formation of the products was confirmed by comparison of their physical and spectral data with authentic samples prepared accordingly to previous methods. Tris(3-indolyl)methanes (5) were also synthesized in similar reaction conditions using 2 equivalents of indoles and 1 equivalents of 3 formylindole (4) in 25 minutes (Scheme 2, Table 2). Compounds 7 and 8 were also synthesized in similar reaction conditions by choosing the appropriate mole ratios of indoles and aldehydes (Scheme 3, Table 2).

\subsection{Spectral Data for Representative Compounds}

3,3'-Bis(indolyl)-4-methoxyphenylmethane (Compound 3b, Table 1): Pinkish solid (yield: $75 \%$ ), M.p. $183^{\circ} \mathrm{C}-184^{\circ} \mathrm{C}$; IR $v_{\max }(\mathrm{KBr}): 3412$ and $3401(\mathrm{~N}-\mathrm{H}), 3050,2950,1217$, $772 \mathrm{~cm}^{-1}$; ${ }^{1} \mathrm{H}$ NMR $\left(\mathrm{CDCl}_{3}, 300 \mathrm{MHz}\right): \delta 3.78(3 \mathrm{H}, \mathrm{s}$, OMe), 5.84 (1H, s, Ar-CH), $6.66(2 \mathrm{H}, \mathrm{s}), 6.81(2 \mathrm{H}, \mathrm{d}, J=$ $8.17 \mathrm{~Hz}), 7.00(2 \mathrm{H}, \mathrm{t}, J=7.5 \mathrm{~Hz}), 7.16(2 \mathrm{H}, \mathrm{t}, J=7.5$ $\mathrm{Hz}), 7.24$ - 7.40 (6H, m), $7.92(2 \mathrm{H}$, br s, NH).

3,3'-Bis(indolyl)-4-benzoyloxy-3-methoxyphenylmethane (Compound 3f, Table 1): White solid (yield: $85 \%$ ), M.p. $238^{\circ} \mathrm{C}-240^{\circ} \mathrm{C}$; IR $v_{\max }(\mathrm{KBr}): 3399$ and 3366 (N-H), 3057, 2966, 1725 (OCOPh), 1276, $743 \mathrm{~cm}^{-1} ;{ }^{1} \mathrm{H}$ NMR $\left(\mathrm{CDCl}_{3}, 300 \mathrm{MHz}\right): \delta 3.68(3 \mathrm{H}, \mathrm{s}, \mathrm{OMe}), 5.90(1 \mathrm{H}$, s, Ar-CH), $6.67(2 \mathrm{H}, \mathrm{s}), 6.91(1 \mathrm{H}, \mathrm{d}, J=8.1 \mathrm{~Hz}), 6.99-$ $7.05(4 \mathrm{H}, \mathrm{m}), 7.17(2 \mathrm{H}, \mathrm{t}, J=7.5 \mathrm{~Hz}), 7.36(2 \mathrm{H}, \mathrm{d}, J=$ 
$8.1 \mathrm{~Hz}), 7.42(2 \mathrm{H}, \mathrm{d}, J=7.8 \mathrm{~Hz}), 7.49(2 \mathrm{H}, \mathrm{t}, J 7.5 \mathrm{~Hz})$, $7.59-7.64(1 \mathrm{H}, \mathrm{m}), 7.94(2 \mathrm{H}, \mathrm{br} \mathrm{s}, \mathrm{NH}), 8.21(2 \mathrm{H}, \mathrm{d}, J=$ $8.1 \mathrm{~Hz}) ; \mathrm{MS}\left(\mathrm{ES}^{+}\right): \mathrm{m} / \mathrm{z}$ Calcd for $\mathrm{C}_{31} \mathrm{H}_{24} \mathrm{~N}_{2} \mathrm{O}_{3}[\mathrm{M}+\mathrm{Na}]^{+}$ 495.17, Found 495.21; Anal. Calcd for $\mathrm{C}_{31} \mathrm{H}_{24} \mathrm{~N}_{2} \mathrm{O}_{3}$ (472.54): C, 78.80; H, 5.12; N, 5.93\%. Found: C, 78.69; $\mathrm{H}, 5.07 ; \mathrm{N}, 5.99 \%$.

3,3'-Bis(indolyl)-4-benzoyloxyphenymethane (Compound 3h, Table 1): White crystalline solid (yield : 88\%), M.p. $216^{\circ} \mathrm{C}$; IR $v_{\max }(\mathrm{KBr}): 3392(\mathrm{~N}-\mathrm{H}), 3062,2950,1723$ (OCOPh), 1270, $745 \mathrm{~cm}^{-1}$; ${ }^{1} \mathrm{H} \mathrm{NMR}\left(\mathrm{CDCl}_{3}, 500 \mathrm{MHz}\right)$ : $\delta 5.92(1 \mathrm{H}, \mathrm{s}, \mathrm{Ar}-\mathrm{CH}), 6.67(2 \mathrm{H}, \mathrm{s}), 7.02(2 \mathrm{H}, \mathrm{t}, J=7.5$ $\mathrm{Hz}), 7.13(2 \mathrm{H}, \mathrm{d}, J=8.5 \mathrm{~Hz}), 7.18(2 \mathrm{H}, \mathrm{t}, J=7.5 \mathrm{~Hz})$, $7.36(2 \mathrm{H}, \mathrm{d}, J=8.0 \mathrm{~Hz}), 7.38(2 \mathrm{H}, \mathrm{d}, J=8.5 \mathrm{~Hz}), 7.39$ $(2 \mathrm{H}, \mathrm{t}, J=8.1 \mathrm{~Hz}), 7.50(2 \mathrm{H}, \mathrm{t}, J=7.8 \mathrm{~Hz}), 7.62(1 \mathrm{H}, \mathrm{m})$, $7.95(2 \mathrm{H}, \mathrm{br} \mathrm{s}, \mathrm{NH}), 8.19(2 \mathrm{H}, \mathrm{d}, J=9.0 \mathrm{~Hz})$; HRMS $\left(\right.$ ES $\left.^{+}\right): \mathrm{m} / \mathrm{z}$ Calcd for $\mathrm{C}_{30} \mathrm{H}_{22} \mathrm{~N}_{2} \mathrm{O}_{2}[\mathrm{M}+\mathrm{Na}]^{+}$465.1578, Found 465.1578 .

3,3'-Bis(2-methylindolyl)-4-benzoyloxy-3-methoxyphen ylmethane (Compound 3v, Table 1): White solid (yield: $90 \%$ ), M.p. $275^{\circ} \mathrm{C}-277^{\circ} \mathrm{C}$; IR $v_{\max }(\mathrm{KBr}): 3401$ and 3375 (N-H), 3057, 2933, 1725 (OCOPh), 1275, 743; ${ }^{1} \mathrm{H}$ NMR (DMSO-d $\left.\mathrm{d}_{6}, 300 \mathrm{MHz}\right): \delta 2.50(6 \mathrm{H}, \mathrm{s}, \mathrm{Me}), 3.55$ $(3 \mathrm{H}, \mathrm{s}, \mathrm{OMe}), 5.97(1 \mathrm{H}, \mathrm{s}, \mathrm{Ar}-\mathrm{CH}), 6.76-6.69(3 \mathrm{H}, \mathrm{m})$, $6.92-6.87(4 \mathrm{H}, \mathrm{m}), 7.10(2 \mathrm{H}, \mathrm{d}, J=9.1 \mathrm{~Hz}), 7.21(2 \mathrm{H}, \mathrm{d}$, $J=8.4 \mathrm{~Hz}), 7.59(2 \mathrm{H}, \mathrm{t}, J=7.5 \mathrm{~Hz}), 7.76-7.71(1 \mathrm{H}, \mathrm{m})$, $8.11(2 \mathrm{H}, \mathrm{d}, J=7.2 \mathrm{~Hz}), 10.76(2 \mathrm{H}, \mathrm{br}$ s, $\mathrm{NH})$; $\mathrm{MS}\left(\mathrm{ES}^{+}\right)$: $\mathrm{m} / \mathrm{z}$ Calcd for $\mathrm{C}_{33} \mathrm{H}_{28} \mathrm{~N}_{2} \mathrm{O}_{3}[\mathrm{M}+\mathrm{Na}]^{+}$523.20, Found 523.26; Anal. Calcd for $\mathrm{C}_{33} \mathrm{H}_{28} \mathrm{~N}_{2} \mathrm{O}_{3}$ (500.60): C, 79.18; H, 5.64; N, 5.60\%. Found: C, 79.56; H, 5.58; N, 5.63\%.

3,3'-Bis(2-methylindolyl)-n-propylmethane (Compound 3x, Table 1): White solid (yield: $73 \%$ ), M.p. $127^{\circ} \mathrm{C}$; IR $v_{\max }(\mathrm{KBr}): 3385(\mathrm{~N}-\mathrm{H}), 3055,2955,1458,1302,745$ $\mathrm{cm}^{-1} ;{ }^{1} \mathrm{H}$ NMR $\left(\mathrm{CDCl}_{3}, 300 \mathrm{MHz}\right): \delta 0.95(3 \mathrm{H}, \mathrm{t}, J=7.5$ $\mathrm{Hz}), 1.36-1.44(2 \mathrm{H}, \mathrm{m}), 2.34(6 \mathrm{H}, \mathrm{s}, \mathrm{Me}), 2.38-2.47$ $(2 \mathrm{H}, \mathrm{m}), 4.41(1 \mathrm{H}, \mathrm{t}, J=7.8 \mathrm{~Hz}, \mathrm{Ar}-\mathrm{CH}), 6.97(2 \mathrm{H}, \mathrm{t}, J=$ $7.5 \mathrm{~Hz}), 7.04(2 \mathrm{H}, \mathrm{t}, J=7.5 \mathrm{~Hz}), 7.21(2 \mathrm{H}, \mathrm{d}, J=8.1 \mathrm{~Hz})$, $7.62(2 \mathrm{H}, \mathrm{d}, J=7.8 \mathrm{~Hz}), 7.64(2 \mathrm{H}, \mathrm{br} \mathrm{s}, \mathrm{NH})$; $\mathrm{MS}\left(\mathrm{ES}^{+}\right)$: $\mathrm{m} / \mathrm{z}$ Calcd for $\mathrm{C}_{22} \mathrm{H}_{24} \mathrm{~N}_{2}[\mathrm{M}+\mathrm{Na}]^{+}$339.18, Found 339.23; Anal. Calcd for $\mathrm{C}_{22} \mathrm{H}_{24} \mathrm{~N}_{2}$ (316.45): C, 83.50; H, 7.64; N, $8.85 \%$. Found: C, 83.41; H, 7.59; N, 8.97\%.

p-3,3'-Bis(2-methylindolyl)methane benzaldehyde (Compound $7 \mathrm{~b}$, Table 2) Pink solid (yield: $72 \%$ ). M.p.: $230^{\circ} \mathrm{C}$ $232^{\circ} \mathrm{C} .{ }^{1} \mathrm{H}$ NMR (300 MHz, DMSO-d 6 ): $\delta 2.08(6 \mathrm{H}, \mathrm{s}$, $\left.\mathrm{CH}_{3}\right), 6.01(1 \mathrm{H}, \mathrm{s}, \mathrm{Ar}-\mathrm{CH}), 6.67(2 \mathrm{H}, \mathrm{t}, J=7.5 \mathrm{~Hz}), 6.79$ $(2 \mathrm{H}, \mathrm{d}, J=7.5 \mathrm{~Hz}), 6.89(2 \mathrm{H}, \mathrm{t}, J=7.8 \mathrm{~Hz}), 7.22(2 \mathrm{H}, \mathrm{d}$, $J=7.8 \mathrm{~Hz}), 7.39(2 \mathrm{H}, \mathrm{d}, J=8.1 \mathrm{~Hz}), 7.81(2 \mathrm{H}, \mathrm{d}, J=8.1$ Hz), 9.96 (1H, s, CHO), 10.82 (2H, br s, NH); MS (ESI) $\mathrm{m} / \mathrm{z}$ : Calcd for $\mathrm{C}_{26} \mathrm{H}_{22} \mathrm{~N}_{2} \mathrm{O}[\mathrm{M}-\mathrm{H}]^{+}$377.17, Found 377.23. Anal. Calcd for $\mathrm{C}_{26} \mathrm{H}_{22} \mathrm{~N}_{2} \mathrm{O}$ (378.47): C, 82.52; H, 5.86; N, 7.40\%. Found: C, 82.62; H, 5.97; N, 7.13\%.

p-Di[3,3'-bis(2-methylindolyl)methane]benzene (Compound 8 b, Table 2) Red solid (yield: $80 \%$ ). M.p.: $282^{\circ} \mathrm{C}$ $285^{\circ} \mathrm{C}$ : IR $v_{\max }(\mathrm{KBr}): 3390(\mathrm{~N}-\mathrm{H}), 3055,2944,1454$, 1300, $746 \mathrm{~cm}^{-1} ;{ }^{1} \mathrm{H}$ NMR (300 MHz, DMSO-d ${ }_{6}$ ): $\delta 2.09$ $\left(12 \mathrm{H}, \mathrm{s}, \mathrm{CH}_{3}\right), 5.87(2 \mathrm{H}, \mathrm{s}), 6.67(4 \mathrm{H}, \mathrm{t}, J=7.5 \mathrm{~Hz}), 6.88$ $(2 \mathrm{H}, \mathrm{t}, J=8.7 \mathrm{~Hz}), 6.90(4 \mathrm{H}, \mathrm{d}, J=8.4 \mathrm{~Hz}), 7.05(4 \mathrm{H}, \mathrm{s})$, $7.18(4 \mathrm{H}, \mathrm{d}, J=7.8 \mathrm{~Hz}), 10.71(4 \mathrm{H}, \mathrm{br} \mathrm{s}, \mathrm{NH}) \cdot{ }^{13} \mathrm{C}$ NMR $\left(75 \mathrm{MHz}, \mathrm{DMSO}-\mathrm{d}_{6}\right): \delta 12.05\left(\mathrm{CH}_{3}\right), 38.26(\mathrm{Ar}-\mathrm{CH})$, $110.29,111.89,112.33,117.84,118.67,19.49,128.29$, 131.99, 135.05, 141.52; MS (ESI) m/z: Calcd for $\mathrm{C}_{44} \mathrm{H}_{38} \mathrm{~N}_{4}[\mathrm{M}-\mathrm{H}]^{+}$621.30, Found 621.25. Anal. Calcd for $\mathrm{C}_{44} \mathrm{H}_{38} \mathrm{~N}_{4}(622.80)$ : C, 84.85; H, 6.15; N, 9.00. Found: C, 84.71; H, 6.27; N, 8.89.

\section{Acknowledgements}

Financial support from the University Grants Commission, New Delhi, Government of India (UGC MRP Grant no. PSW-130/11-12-ERO) is gratefully acknowledged.

\section{REFERENCES}

[1] R. J. Sundberg, "The Chemistry of Indoles," Academic Press, New York, 1996.

[2] M. Shiri, M. A. Zolfigol, H. G. Kruger and Z. Tanbakouchian, "Bis- and Trisindolylmethanes (BIMs and TIMs)," Chemical Reviews, Vol. 110, No. 4, 2010, pp. 2250-2293. doi:10.1021/cr900195a

[3] C. A. Bradfield and L. F. Bjeldanes, "Structure-Activity Relationships of Dietary Indoles: A Proposed Mechanism of Action as Modifiers of Xenobiotic Metabolism," Journal of Toxicology and Environmental Health, Vol. 21, No. 3, 2003, pp. 311-323. doi:10.1080/15287398709531021

[4] M. Chakrabarty, N. Ghosh, R. Basak and Y. Harigaya, "Dry Reaction of Indoles with Carbonyl Compounds on Montmorillonite K10 Clay: A Mild, Expedient Synthesis of Diindolylalkanes and Vibrindole A," Tetrahedron Letters, Vol. 43, No. 22, 2002, pp. 4075-4078. doi:10.1016/S0040-4039(02)00682-2

[5] A. K. Mallik, R. Pal and T. K. Mandal, "Facile Formation of Bis(3-indolyl)methylarenes by Iodine-Catalyzed Reaction of Indole with $\alpha, \alpha^{\prime}$-Bis(arylmethylene)ketones and $\alpha$-Substituted Arylmethyleneketones in Dry Ethanol," Indian Journal of Chemistry, Vol. 46B, No. 12, 2007, pp. 2056-2059.

[6] R. J. Sundberg, "The Chemistry of Indoles," Academic Press, New York, 1970.

[7] M. Roomi and S. MacDonald, "Reductive C-Alkylation II," Canadian Journal of Chemistry, Vol. 48, No. 1, 1970, pp. 39-143. doi:10.1139/v70-019

[8] D. M. Pore, U. V. Desai, T. S. Thopate and P. P. Wadgaonkar, "A Mild, Expedient, Solventless Synthesis of Bis(indolyl)alkanes Using Silica Sulfuric Acid as a Reusable Catalyst," Arkivoc, Vol. xii, 2006, pp. 75-80.

doi:10.3998/ark.5550190.0007.c09

[9] C. Ramesh, J. Banerjee, R. Pal and B. Das, "Silica Supported Sodium Hydrogen Sulfate and Amberlyst-15: Two Efficient Heterogeneous Catalysts for Facile Synthesis of Bis- and Tris(1H-indol-3-yl)methanes from Indoles and Carbonyl Compounds," Advanced Synthesis \& Catalysis, Vol. 345, No. 3, 2003, pp. 557-559.

doi:10.1002/adsc.200303022 
[10] K. R. M. Naidu, S. I. Khalivulla, P. C. R. Kumar and O. Lasekan, "KHSO $4-\mathrm{SiO}_{2}$ Catalyzed Facile Synthesis of Bis(indolyl)methanes," Organic Communications, Vol. 5, No. 3, 2012, pp. 150-159.

[11] J. T. Li and S. F. Sun, "Synthesis of Diindolylmethanes (DIMs) Catalyzed by Sillicotungstic Acid by Grinding Method," E-Journal of Chemistry, Vol. 7, No. 3, 2010, pp. 922-926. doi:10.1155/2010/986980

[12] G. A. Meshram and V. D. Patil, "Simple and Efficient Method for Synthesis of Bis(indolyl) Methanes with $\mathrm{Cu}\left(\mathrm{BF}_{4}\right)_{2} \cdot \mathrm{SiO}_{2}$ Under Mild Conditions," Synthetic Communications, Vol. 40, No. 1, 2010, pp. 29-38. doi:10.1080/00397910902916064

[13] J. S. Yadav, B. V. S. Reddy, C. V. S. R. Murthy, G. M. Kumar and C. Madan, "Lithium Perchlorate Catalyzed Reactions of Indoles: An Expeditious Synthesis of Bis(indolyl)methanes," Synthesis, Vol. 5, 2001, pp. 783787. doi:10.1055/s-2001-12777

[14] R. Nagarajan and P. T. Perumal, " $\mathrm{InCl}_{3}$ and $\mathrm{In}(\mathrm{OTf})_{3}$ Catalyzed Reaction: Synthesis of 3-Acetyl Indoles, BisIndolylmethanes and Indolylquinoline Derivatives," Tetrahedron, Vol. 58, No. 6, 2002, pp. 1229-1232. doi:10.1016/S0040-4020(01)01227-3

[15] S. J. Ji, S. Y. Wang, Y. Zhang and T. P. Loh, "Facile Synthesis of Bis(indolyl)methanes Using Catalytic Amount of Iodine at Room Temperature under Solvent-Free Conditions," Tetrahedron, Vol. 60, No. 9, 2004, pp. 20512055. doi:10.1016/j.tet.2003.12.060

[16] G. V. M. Sharma, J. J. Reddy, P. S. Lakshmi and R. P. Krishna, "A Versatile and Practical Synthesis of Bis(indolyl)methanes/Bis(indolyl)glycoconjugates Catalyzed by Trichloro-1,3,5-triazine," Tetrahedron Letters, Vol. 45, No. 41, 2004, pp. 7729-7732. doi:10.1016/i.tetlet.2004.08.084

[17] R. Nagarajan and P. T. Perumal, "Electrophilic Substitution of Indoles Catalyzed by Triphenyl Phosphonium Trichlorate: Synthesis of 3-Acetyl Indoles and Bis-Indolylmethane Derivatives," Synthetic Communications, Vol. 32, No. 1, 2002, pp. 105-109. doi:10.1081/SCC-120001515

[18] C. J. Li and T. H. Chan, "Organic Reactions in Aqueous Media," John Wiley \& Sons, New York, 1997.

[19] A. Kamal and A. A. Qureshi, "Syntheses of Some Substituted Di-indolylmethanes in Aqueous Medium at Room Temperature," Tetrahedron, Vol. 19, No. 4, 1963, pp. 513-520. doi:10.1016/S0040-4020(01)98540-0

[20] S. Kumar, I. S. Grover and J. S. Sandhu, "A Practical, Clean and Green Synthesis of Vibrindole and Bis(indolyl)methanes Catalyzed by Alum $\left[\mathrm{KAl}\left(\mathrm{SO}_{4}\right)_{2} \cdot 12 \mathrm{H}_{2} \mathrm{O}\right]$ in Water," Indian Journal of Chemistry, Vol. 48B, No. 4, 2009, pp. 585-589.

[21] A. K. Mallik, R. Pal, C. Guha and H. Mallik, "A Convenient, Eco-friendly, and Efficient Method for Synthesis of Bis(3-indolyl)methanes on Water," Green Chemistry Letters and Reviews, Vol. 5, No. 3, 2012, pp. 321-327. doi:10.1080/17518253.2011.630027

[22] D. Chen, L. Yu and P.-G. Wang, "Lewis Acid-Catalyzed Reactions in Protic Media: Lanthanide-Catalyzed Reactions of Indoles with Aldehydes or Ketones," Tetrahe- dron Letters, Vol. 37, No. 26, 1996, pp. 4467-4470. doi:10.1016/0040-4039(96)00958-6

[23] S. Mishra and R. Ghosh, "Ecofriendly and Sustainable Efficient Synthesis of Bis(indolyl)methanes Based on Recyclable Brønsted (CSA) or Lewis $\left(\mathrm{ZrOCl}_{2} \cdot 8 \mathrm{H}_{2} \mathrm{O}\right)$ Acid Catalysts," Indian Journal of Chemistry, Vol. 50B, No. 11, 2011, pp. 1630-1636.

[24] M. L. Deb and P. J. Bhuyan, "An Efficient and Clean Synthesis of Bis(indolyl)methanes in a Protic Solvent at Room Temperature," Tetrahedron Letters, Vol. 47, No. 9, 2006, pp. 1441-1443. doi:10.1016/i.tetlet.2005.12.093

[25] R. Ghorbani-Vaghei, H. Veisi, H. Keypour and A. A. Dehghani-Firouzabadi, "A Practical and Efficient Synthesis of Bis(indolyl)methanes in Water, and Synthesis of Di-, Tri-, and Tetra(bis-indolyl)methanes under Thermal Conditions Catalyzed by Oxalic Acid Dihydrate," Molecular Diversity, Vol. 14, No. 1, 2010, pp. 87-96. doi:10.1007/s11030-009-9150-Z

[26] S. Y. Wang and S. J. Ji, "Facile Synthesis of Bis(indolyl)methanes Catalyzed by Ferric Dodecyl Sulfonate $\left[\mathrm{Fe}(\mathrm{DS})_{3}\right]$ in Water at Room Temperature," Synthetic Communications, Vol. 38, No. 8, 2008, pp. 1291-1298. doi:10.1080/00397910701873318

[27] T. J. Mason, "Practical Sonochemistry," Ellis Horwood, New York, 1991.

[28] J. T. Li, S. X. Wang, G. F. Chen and T. S. Li, "Some Applications of Ultrasound Irradiation in Organic Syn-thesis," Current Organic Synthesis, Vol. 2, No. 3, 2005, pp. 415-436. doi:10.2174/1570179054368509

[29] X. F. Zeng, S. J. Ji and S. Y. Wang, "Novel Method for Syntheis of Unsymmetrical Bis(indolyl)alkanes Catalyzed by Cerric Ammonium Nitrate (CAN) under Ultrasonic Irradiation," Tetrahedron, Vol. 61, No. 43, 2005, pp. 10235-10241. doi:10.1016/i.tet.2005.08.040

[30] J. T. Li, H. G. Dai, W. Z. Xu and T. S. Li, “An Effficient and Practical Synthesis of Bis(indolyl)methanes Catalyzed by Aminosulfonic Acid Under Ultrasound," Ultrasonics Sonochemistry, Vol. 13, No. 1, 2006, pp. 24-27. doi:10.1016/j.ultsonch.2004.12.004

[31] S. Y. Wang, S. J. Ji and X. M. Su, "A Meldrum's Catalyzed Bis(indolyl)methanes in Water Under Ultrasonic Condition," Chinese Journal of Chemistry, Vol. 26, No. 1, 2008, pp. 22-24.

[32] J. T. Li, X. H. Zhang and Y. L. Song, "Efficient Synthesis of Bis(indolyl)methanes Catalyzed by Silicotungstic Acid Under Ultrasound Irradiation," International Journal of Chem Tech Research, Vol. 2, No. 1, 2010, pp. 341-345. doi:10.1002/cjoc.200890029

[33] J. T. Li, M. X. Sun, G. Y. He and X. Y. Xu, "Efficient and Green Synthesis of Bis(indolyl)methanes Catalyzed by ABS in Aqueous Media Under Ultrasound Irradiation," Ultrasonics Sonochemistry, Vol. 18, No. 1, 2011, pp. 412-414. doi:10.1016/j.ultsonch.2010.07.016

[34] R. Pal, S. Khasnobis and T. Sarkar, "First Application of Fruit Juice of Citrus Limon for Facile and Green Synthesis of Bis- and Tris(indolyl)methanes in Water," Chemistry Journal, Vol. 3, No. 1, 2013, pp. 7-12.

[35] R. Pal, "Microwave-assisted Eco-Friendly Synthesis of 
Bis-, Tris(indolyl)methanes and Synthesis of Di-bis(indolyl)methanes Catalyzed by Fruit Juice of Citrus Limon Under Solvent-free Conditions," IOSR Journal of Applied
Chemistry, Vol. 3, No. 4, 2013, pp. 1-8. doi: $10.9790 / 5736-0340108$ 